\title{
Long Noncoding RNA Meg3 Regulates Mafa Expression in Mouse Beta Cells by Inactivating Rad21, Smc3 or Sin3 $\alpha$
}

\author{
Ning Wang ${ }^{\mathrm{a}}$ Yanan Zhu ${ }^{\mathrm{a}} \quad$ Min Xie $^{\mathrm{a}}$ Lintao Wang ${ }^{\mathrm{a}}$ Feiyan Jin ${ }^{\mathrm{a}}$ Yihui Li ${ }^{\mathrm{b}}$ \\ Qingxin Yuan ${ }^{b}$ Wei De
}

aDepartment of Biochemistry and Molecular Biology, Nanjing Medical University, Nanjing, bepartment of Endocrinology, First Affiliated Hospital of Nanjing Medical University, Nanjing, PR China

\author{
Key Words \\ Long noncoding RNA $\cdot$ Meg3 $\cdot$ Beta cells $\bullet$ MafA $\cdot \operatorname{Rad} 21 \cdot \operatorname{Smc} 3 \cdot \operatorname{Sin} 3 \alpha$
}

\begin{abstract}
Background/Aims: The main pathogenic mechanism of diabetes is a decrease in the number of islet beta cells or a decline in their function. Recent studies have shown that pancreatic long noncoding RNAs (IncRNAs) have a high degree of tissue specificity and may be involved in the maintenance of islet cells function and the development of diabetes. The aim of this study was to investigate the molecular regulatory mechanism of mouse maternal expressed gene 3 (Meg3) in insulin biosynthesis in pancreatic islets. Methods: Chromatin immunoprecipitationquantitative polymerase chain reaction ( $q P C R$ ) and RNA immunoprecipitation-qPCR were used to investigate the molecular mechanism of IncRNA Meg3 in insulin biosynthesis by regulating $v$-Maf musculoaponeurotic fibrosarcoma oncogene family, protein A (MafA), a mature beta cell marker in the MIN6 beta cell line. Further, the expression levels of Meg3, Ezh2, MafA, Rad21, Smc3, and Sin3 $\alpha$ were analyzed in vivo and in vitro by RT-PCR and western blotting. Results: Intranuclear IncRNA Meg3 can bind EZH2, a methyltransferase belonging to the Polycomb repressive complex-2, in pancreatic islet cells. In addition, knockdown of Ezh2 can also inhibit the expression of MafA and Ins2, while expression levels of Rad21, Smc3, and $\operatorname{Sin} 3 \alpha$ are upregulated, by interfering with Ezh2 or Meg3 in pancreatic beta cells. Knockdown of Meg3 resulted in the loss of EZH2 binding and H3K27 trimethylation occupancy of Rad21, $S m c 3$, and $\operatorname{Sin} 3 \alpha$ promoter regions. The inhibition of $R a d 21, \operatorname{Smc} 3$, or $\operatorname{Sin} 3 \alpha$, which directly act on the MafA promoter, leads to upregulated expression of MafA in both MIN6 cells and mouse islets. Moreover, the synthesis and secretion of insulin were increased by inhibition of these transcription factors. Conclusions: Pancreatic IncRNA Meg3 can epigenetically regulate the expression of Rad21, Smc3, and $\operatorname{Sin} 3 \alpha$ via EZH2-driven H3K27 methylation. By inhibiting the expression of Rad21, Smc3, or $\operatorname{Sin} 3 \alpha$, Meg3 promotes the expression of MafA and affects the production of insulin.

Department of Biochemistry and Molecular Biology, Nanjing Medical University, Nanjing, Department of Endocrinology, First Affiliated Hospital of Nanjing Medical University, Nanjing (PR China),E-Mail dewei@njmu.edu.cn, yuanqingxin@vip.163.com 


\section{Cellular Physiology Cell Physiol Biochem 2018;45:2031-2043 and Biochemistry Published / \begin{tabular}{l|l} 
DOI: 10.1159/000487983 & $\begin{array}{l}\text { C } 2018 \text { The Author(s). Published by S. Karger AG, Basel } \\
\text { www.karger.com/cpb }\end{array}$
\end{tabular} \\ Wang et al.: Pancreatic Meg3 Regulates MafA by Inactivating Rad21, Smc3 or $\sin 3 \alpha$}

\section{Introduction}

Synthesis and secretion of insulin are the main functions of pancreatic beta cells. Beta cell dysfunction or mass reduction appears in almost all types of diabetes mellitus and thus eventually leads to insulin deficiency [1]. The biosynthesis of insulin is regulated by many transcription factors, such as v-Maf musculoaponeurotic fibrosarcoma, oncogene family, protein A (MafA) [2-5], neurogenic differentiation1 (NeuroD1) [6, 7], and pancreatic and duodenal homeobox 1 (PDX1) [8, 9]. The transcriptional activator MafA, which regulates insulin transcription in response to circulating glucose levels, is expressed in mature pancreatic beta cells $[2,3,10,11]$. It is essential for the maintenance of islet structure and function [12] and the expression of MafA is regulated by long noncoding RNAs (lncRNAs) $[13,14]$. Studies have shown that expression of IncRNAs may influence susceptibility to diabetes $[15,16]$. IncRNAs, located in the nucleus or cytoplasm, are generally more than 200 nucleotides and do not encode a functional protein. They regulate a number of biological processes, such as cell proliferation and apoptosis. Notably, studies have demonstrated that lncRNAs are also implicated in many human diseases [17-19]. Ferrer et al. found human pancreatic lncRNAs with high tissue specificity. Depletion of the beta cell-specific lncRNA HI-LNC25 may significantly downregulate the expression of the diabetes susceptibility gene GLIS3 [16], which increases the risk of both type 1 and type 2 diabetes [20]. The lncRNAs maternally expressed gene 3 (Meg3) [13] and taurine upregulated gene 1(TUG1) [14] are associated with the maintenance of pancreatic beta cell function.

MEG3 is located on human chromosome 14q32.3 and encodes an lncRNA. In the mouse, it is also known as gene trap locus 2 (Gtl2), and is located at the distal chromosome 12 [21, 22]. Microarray analysis indicates that MEG3 expression is 20 -fold higher in human beta cells compared with alpha cells [23] and is downregulated in type 2 diabetes donor islets [15]. belonging to the imprinted DLK1-MEG3 gene region, has strong homology in humans and mice. RNA expressions from the DLK1-MEG3 locus are determined by the methylation of two differentially methylated regions (DMRs), "IG-DMR" and "MEG3-DMR". The "MEG3-DMR" is located $1.5 \mathrm{~kb}$ upstream of the transcription initiation site of $M E G 3$, and overlaps with the MEG3 promoter. Methylation of this region is increased in type 2 diabetes islets [15]. Further, genome-wide association studies revealed that this region alters susceptibility to type 1 diabetes [24]. In addition, we found that Meg3 was downregulated in pancreatic islets of type 1 (NOD) and type $2(\mathrm{db} / \mathrm{db}$ ) diabetic mice. All of these findings show that lncRNA Meg3 is essential for the proper function of pancreatic islets. In MIN6 cells and mouse pancreatic islets, Meg3 expression is dynamically regulated by glucose, and the suppression of Meg3 leads to reduced expression of the transcription factors MafA [13], although the molecular mechanisms remain unknown.

In this study, we demonstrated the mechanism of MafA regulation by lncRNA Meg3 in mouse pancreatic beta cells. Through bioinformatics analysis, we found the inhibitory factors RAD21 cohesin complex component (Rad21), structural maintenance of chromosomes 3 (Smc3), and transcriptional regulator SIN3A (yeast) (Sin3 $\alpha$ ), which bind to the promoter of MafA. Further, we examined the role of Meg3 in the regulation of these inhibitory factors through EZH2. These findings improve understanding of the mechanism of insulin biosynthesis and provide novel insight into the pathogenesis of diabetes.

\section{Materials and Methods}

\section{Islet isolation and culture}

The Ethics Committee for the use of Experimental Animals at Nanjing Medical University approved all the animal experiments. Mouse pancreatic islets were isolated, handpicked as previously described [25] from NOD male mice, db/db male mice (Model Animal Research Center of NJU, Nanjing, Jiangsu, China), Balb/c male mice, and C57BL/6J male mice(8 weeks old; 20-23 g; Experimental Animal Center of Nanjing Medical University, Nanjing, Jiangsu, China). 


\section{Cellular Physiology and Biochemistry}

Cell Physiol Biochem 2018;45:2031-2043

\begin{tabular}{l|l}
\hline DOI: $10.1159 / 000487983$ & (c) 2018 The Author(s). Published by S. Karger AG, Basel
\end{tabular}

Published online: March 09, 2018 www.karger.com/cpb

Wang et al.: Pancreatic Meg3 Regulates MafA by Inactivating Rad21, Smc3 or $\operatorname{Sin} 3 \alpha$

Pancreatic islets were cultured with complete RPMI-1640 medium (Invitrogen, Carlsbad, CA, USA), supplemented with $10 \%$ fetal bovine serum (FBS), $100 \mathrm{U} / \mathrm{mL}$ penicillin, and $100 \mathrm{mg} / \mathrm{mL}$ streptomycin (Invitrogen).

\section{MIN6 cell culture}

The murine insulin-secreting cell line Min6 was cultured in DMEM (Invitrogen) containing 25 mM D-glucose, $10 \mathrm{mM}$ HEPES , $50 \mathrm{mM}$ sodium pyruvate, supplemented with 15\% FBS, $100 \mathrm{U} /$ $\mathrm{mL}$ penicillin, $100 \mathrm{mg} / \mathrm{mL}$ streptomycin, and 50 mmol/L $\beta$-mercaptoethanol (Sigma-Aldrich, St. Louis, MO, USA). Cells were planted at a density of $5 \times 10^{5} / \mathrm{cm}^{2}$ for RNA isolation and $10^{5} / \mathrm{cm}^{2}$ for insulin secretion.

\section{RNA interference in vitro and in vivo}

Small interfering (si) RNA was synthesized from Realgene and transfected to cells for $48 \mathrm{~h}$ by InstantFECT transfection reagent (PGR-Solutions Inc., Bridgeville, PA, USA). The mock group was set by cells cultured with the transfection reagent alone, and scrambled siRNA was used as negative control (si-NC). According to the instruction manual, the final concentration of siRNA was $100 \mathrm{nmol} / \mathrm{L}$. siRNA delivery in vivo by hydrodynamic injection has been described in our recent study [26]. The siRNA sequences are listed in Table 1.

\section{Quantitative RT-PCR ( $q$ RT-PCR) analysis}

Total RNA was extracted using TRIzol reagent (Invitrogen) from mouse islets and MIN6 cells. RNA $(1 \mu \mathrm{g})$ was reverse-transcribed to cDNA using a Reverse Transcription Kit (Takara, Dalian, China). Quantitative reverse transcription-polymerase chain reaction (qRTPCR) analyses were performed using SYBR Premix Ex Taq (Takara). Gene expression was determined with normalization to mouse $18 \mathrm{~S}$ ribosomal RNA expression by $2^{-\Delta \Delta C t}$ methodology. The primer sequences are listed in Table 1.

\section{Subcellular fractionation location}

According to the manufacturer's instructions, nucleocytoplasmic separation was performed using the PARIS Kit (Life Technologies).

\section{Western blot analysis}

After determining the protein content of mouse islets and Min6 cells, Western blotting was performed as described previously [27]. The blot was probed with antibody: anti-MafA (sc-66958, Santa Cruz Biotechnology, CA), anti-Rad21 (ab992, Abcam), anti-SMC3 (ab9263, Abcam), anti-Sin3 $\alpha$ (ab3479, Abcam), and anti- $\beta$-Actin (\#4970, Cell Signaling Technology, MA). The secondary antibody was from Beijing Zhong Shan Biotechnology CO (Beijing, China).
Table 1. Primers for RT-qPCR and ChIP-qPCR, and mouse siRNA sequences for transfection to cells

\begin{tabular}{|c|c|}
\hline Primer name & Sequence( RT-qPCR) \\
\hline Meg3 forward & 5'-GGACTTCACGCACAACACGTT-3' \\
\hline Meg3 reverse & 5'-GTCCCACGCAGGATTCCA-3' \\
\hline Ezh2 forward & 5'-GCCAGACTGGGAAGAAATCTG-3' \\
\hline Ezh2 reverse & 5'-TGTGCTGGAAAATCCAAG-3' \\
\hline Ins2 forward & 5'-TCCGCTACAATCAAAAACCAT-3' \\
\hline Ins2 reverse & 5'-GCTGGGTAGTGGTGGGTCTA-3' \\
\hline MafA forward & 5'-ATCATCACTCTGCCCACCAT-3' \\
\hline MafA reverse & 5'-AGTCGGATGACCTCCTCCTT-3' \\
\hline Rad21 forward & 5'-ATGTTCTACGCACATTTT GTCCT-3' \\
\hline Rad 21 reverse & 5'-CATGGGCTTTGGTTAGCTTCT-3' \\
\hline Smc3 forward & 5 '-TCCAGGGCTTCCGAAGTTAC-3' \\
\hline Smc3 reverse & 5'-TTCTGCCCACGATGACATTATG-3' \\
\hline Sin $3 \alpha$ forward & 5'-ATGAAGCGACGTTTGGATGAC-3' \\
\hline $\operatorname{Sin} 3 \alpha$ reverse & 5'-CTGAAACCTGGTAGTTGGGTG-3' \\
\hline Ctcf forward & 5'-GATCCTACCCTTCTCCAGATGAA-3' \\
\hline Ctcf reverse & 5'-GTACCGTCACAGGAACAGGT-3' \\
\hline Ets 1 forward & 5'-GATCTCAAGCCGACTCTCACC-3' \\
\hline Ets 1 reverse & 5 '-GACGTGGGTTTCTGTCCACT-3' \\
\hline Maz forward & 5'-GCCCCAGTTGCATCTGTCTT-3' \\
\hline Maz reverse & 5'-GGAGGTTGTAGCCGTTCTTGA-3' \\
\hline Mxi1 forward & 5'-CACAATGAGTTGGAAAAGAACCG-3' \\
\hline Mxi1 reverse & 5'-CCAGCGGGATCAGAACTTTCA-3' \\
\hline Nelfe forward & 5 '-CGGTGTCAAACGCTCACTGT-3' \\
\hline Nelfe reverse & 5'-GCCTGAGTTCTTAGTTTCAGCCT-3' \\
\hline Tbp forward & 5'-ATGATGCCTTACGGCACAGG-3' \\
\hline Tbp reverse & 5'-GTTGCTGAGATGTTGATTGCTG-3' \\
\hline E2f4 forward & 5'-CTCACCACCAAGTTCGTGTC-3' \\
\hline E2f4 reverse & 5'-TCTCGATCAGACCGATGCCTT-3' \\
\hline Tef 12 forward & 5'-ATGTACTGTGCTTATCCTGTCCC-3' \\
\hline Tcf 12 reverse & 5'-GGTGCATATACCGTTTTCCCATT-3' \\
\hline Tcf3 forward & 5'-TTTGACCCTAGCCGGACATAC-3' \\
\hline Tcf3 reverse & 5'-GCATAGGCATTCCGCTCAC-3' \\
\hline 18S rRNA forward & 5'-GTAACCCGTTGAACCCCATT-3' \\
\hline $18 \mathrm{~S}$ rRNA reverse & 5'-CCATCCAATCGGTAGTAGCG-3' \\
\hline gapdh forward & 5'-GACTCATGACCACAGTCCATGC-3' \\
\hline gapdh reverse & 5'-AGAGGCAGGGATGATGTTCTG-3' \\
\hline Primer name & Sequence(ChIP-qPCR) \\
\hline MafA forward & 5'-CATTCCAATGGCTCCCCGAA-3' \\
\hline MafA reverse & 5'-CAGCTCCTTCCCTCAGTGTC-3' \\
\hline Rad21 forward & 5'-ACAGCAAGCAGTTTCTCGGT-3' \\
\hline & 5'-CTGAAGGTGCGTGTGTCAAC-3' \\
\hline Smc3 forward & 5 '-ACAAGGTGAAATCGGGGCTT-3' \\
\hline Smc3 reverse & 5'-ACTGGAGGACGCTAGGACAT-3' \\
\hline $\operatorname{Sin} 3 \alpha$ forward & 5'-CCATAGCGGCTCAGACAAGT-3' \\
\hline Sin $3 \alpha$ reverse & 5'-GCCTGGCTAGCATTTGGTTG-3' \\
\hline Hoxa9 forward & 5'-AAAAGTCAAATACAAGGCTCTCTCC-3' \\
\hline Hoxa9 reverse & 5'-CCAGTCTGACTAGGGTCGGTAG-3' \\
\hline Primer name & Sequence \\
\hline si-Meg3-1 sense & 5'-AUUGGAGGUGAGGAAGGAAAGCAGC-3' \\
\hline $\begin{array}{l}\text { Si-Meg3-1 antise } \\
\text { si-Meg }\end{array}$ & 5'-GCUGCUUUCCUUCCUCACCUCCAAU-3' \\
\hline $\begin{array}{l}\text { si-Meg3-2 sense } \\
\text { si-Men }\end{array}$ & 5'-AACAGCAAAUGGCACAGGAAGAGACGC-3' \\
\hline si-Meg3-2 antisense & 5'-GCGUCUUCCUGUGCCAUUUGCUGUU-3' \\
\hline si-Ezh2-1 sense & 5'-GACCACAGGAUAGGCAUCUUUGCUA-3' \\
\hline si-Ezh2-1 antisense & 5'-UAGCAAAGAUGCCUAUCCUGUGGUC-3' \\
\hline si-Ezh2-2 sense & 5 '-UCAUGUACUCUGAUUUUACAC-3' \\
\hline si-Ezh $2-2$ antisense & 5 '-GUAAAAUCAGAGUACAUGAGA-3' \\
\hline si-Rad21-1 sense & 5'-GGAAUAACCGGCUACUGAATT-3' \\
\hline si-Rad21-1 antisense & 5'-UUCAGUAGCCGGUUAUUCCTT-3' \\
\hline $\begin{array}{l}\text { Si-Rad21-1 antisense } \\
\text { si-Rad21-2 sense }\end{array}$ & 5'-CCGCAUCUAUCACAGGAAATT-3' \\
\hline si-Rad21-2 anti & 5'-UUUCCUGUGAUAGAUGCGGTT-3' \\
\hline si-Smc3-1 sense & 5'-GGCAGAAACAGAACCUAAATT-3' \\
\hline si-Smc3-1 antisense & 5 '-UUUAGGUUCUGUUUCUGCCTT-3' \\
\hline si-Smc3-2 sense & 5'-GGACCAAGUUGGAGCUUAATT-3' \\
\hline si-Smc3-2 antisense & 5'-UUAAGCUCCAACUUGGUCCTT-3' \\
\hline si-Sin $3 \alpha-1$ sense & 5'-CCGAGUGUCCCAGCUAUUUTT-3' \\
\hline si-Sin $3 \alpha-1$ antisense & 5'-AAAUAGCUGGGACACUCGGTT-3' \\
\hline si-Sin $3 \alpha-2$ sense & 5'-CCUCAGGUCUACAAUGAUUTT-3' \\
\hline si-Sin $3 \alpha-2$ antise & 5'-AAUCAUUGUAGACCUGAGGTT-3' \\
\hline si & 5'-GACUU \\
\hline si-MafA-1 antisense & 5'-ACUUCAUCAGGUCGAAGUCTT-3' \\
\hline si-MafA-2 sense & 5'-GGACCUGUACAAGGAGAAAUA-3' \\
\hline si-MafA- 2 antisense & 5 '-UUUCUCCUUGUACAGGUCCCG-3' \\
\hline
\end{tabular}




\section{Cellular Physiology Cell Physiol Biochem 2018;45:2031-2043 \begin{tabular}{l|l} 
and Biochemistry Published online: March 09, 2018 & $\begin{array}{l}\text { (c) } 2018 \text { The Author(s). Published by S. Karger AG, Basel } \\
\text { www.karger.com/cpb }\end{array}$
\end{tabular}

\section{Chromatin immunoprecipitation (ChIP)}

According to the manufacturer's instruction, ChIP assays were performed using an EZ-ChIP kit (Millipore, USA). For immunoprecipitation, $5 \mu \mathrm{g}$ of rabbit antibody anti-Rad21 (ab992, Abcam), anti-SMC3 (ab9263, Abcam), anti-Sin3 $\alpha$ (ab3479, Abcam), anti-EZH2 (\#5246, Cell Signaling Technology), H3 trimethyl Lys 27 antibody (Millipore, USA), or normal rabbit IgG (Millipore) was used, and DNA quantification was performed by qPCR with SYBR Green Mix (Takara). All primers for genes promoters are described in Table 1.

\section{RNA immunoprecipitation (RIP)}

According to the manufacturer's instructions, RIP assays were performed using Magna RIP ${ }^{\text {TM }}$ RNABinding Protein Immunoprecipitation Kit (Millipore) and $5 \mu \mathrm{g}$ of the antibody anti-EZH2 (no.17-662, Millipore).

Glucose stimulated insulin secretion (GSIS)

Min6 cells were planted in 24-well plates at $10^{5}$ per well. At $48 \mathrm{~h}$ posttransfection, the medium was removed and cells were washed twice with sterile Krebs Ringer buffer (KRB) (115 mM NaCl, 4.7 mM KCl, 1.2 $\mathrm{mM} \mathrm{MgSO}_{4}, 1.2 \mathrm{mM} \mathrm{KH}_{2} \mathrm{PO}_{4}, 20 \mathrm{mM} \mathrm{NaHCO}_{3}, 16 \mathrm{mM} \mathrm{HEPES}, 2.56 \mathrm{mM} \mathrm{CaCl}_{2}$, and $0.2 \%$ bovine serum albumin [BSA]) followed by pre-incubation with $200 \mathrm{ml}$ of the same buffer supplemented with $2 \mathrm{mM}$ glucose at $37^{\circ} \mathrm{C}$ for $1 \mathrm{~h}$. Subsequently, the cells were treated for $1 \mathrm{~h}$ in $200 \mu \mathrm{l}$ KRB buffer containing with low (2 $\mathrm{mM})$ or high $(20 \mathrm{mM})$ concentrations of glucose. Supernatants were collected at the indicated time and insulin concentrations were determined using radioimmunoassay (RIA) as previously described [28]. After transfection for $48 \mathrm{~h}$ (at $25 \mathrm{mM}$ glucose), cellular insulin content was detected after acid ethanol extraction $(0.15 \mathrm{M} \mathrm{HCl}$ in $75 \%$ ethanol). These results were normalized to the total protein concentration as previously described $[13,29,30]$. Subsequently, the amount of secreted insulin protein was counted (as a ratio) in comparison with the total cellular insulin content in the experiment.

\section{Statistical analysis}

The data are presented as mean \pm SEM. Statistical significances $(p<0.05)$, which are indicated in the figures, were performed using a two-tailed paired Student's $t$-test, or analysis of variance (ANOVA) for more than two groups comparison (GraphPad Prism 5, San Diego, California, USA).

\section{Results}

IncRNA Meg3 regulates expression of MafA through EZH2 in pancreatic beta cells

To investigate the possible mechanism of lncRNA Meg3 regulation of MafA in beta cells, it was necessary to define the intracellular localization of Meg3. Meg3 expression was measured in nuclear and cytosolic fractions from MIN6 cells by qRT-PCR, and the differential enrichments of $U 1$ and Gapdh RNA were used as fractionation indicators. We found a considerable increase in the expression of Meg3 in the nucleus relative to the cytosol (Fig. 1A), which suggested that Meg3 is primarily localized in the nucleus and plays a regulatory role in the levels of transcription. Numerous lncRNAs (HOTAIR, MALAT1, EBIC, H19, ANRIL, etc.) have been confirmed to regulate the expression of target genes via EZH2-driven H3K27 methylation [31-34]. EZH2, a methyltransferase belonging to the core component of the Polycomb repressive complex-2 (PRC2), is responsible for the trimethylation of lysine 27 in histone H3 (H3K27me3) [35]. EZH2 photoactivatable-ribonucleotide-enhanced crosslinking and immunoprecipitation (PAR-CLIP) data [36] revealed that EZH2 interacted with 165 lncRNAs, including Meg3, in mouse embryonic stem cells (mESCs) [37, 38]. Therefore, we examined whether the lncRNA Meg3 plays a role in islet beta cells by binding to EZH2. As shown in Fig. 1B, endogenous Meg3 was enriched in the anti-EZH2 RNA immunoprecipitation (RIP) fraction relative to the IgG fraction in MIN6 cells. Endogenous Gapdh was used as a negative control. Thus, lncRNA Meg3 plays a role in pancreatic beta cells as well as in mESCs by binding to EZH2. Moreover, we transfected siRNA to knockdown Ezh2 in both MIN6 cells and pancreatic islets. The knockdown efficiency of Ezh2 is shown in Fig. 1C; the si-Ezh2 group was a mixture of two interfering sequences. Because the interference efficiency of 


\section{Cellular Physiology Cell Physiol Biochem 2018;45:2031-2043 and Biochemistry \begin{tabular}{c|c} 
DOI: 10.1159/000487983 & $\begin{array}{l}\text { O 2018 The Author(s). Published by S. Karger AG, Basel } \\
\text { www.karger.com/cpb }\end{array}$
\end{tabular}

Fig. 1. IncRNA Meg3 regulates expression of MafA through EZH2 in pancreatic beta cells. (A) Meg3 expression levels in the cell nucleus or cytoplasm of MIN6 cells were detected by qRT-PCR. U6 was used as a nucleus marker, and Gapdh was used as a cytosol marker. (B) RIP experiments were performed in MIN6 cells, and the coprecipitated RNA was subjected to qRT-PCR for Meg3. The fold enrichment of Meg3 in EZH2 RIP was relative to its matching IgG control RIP. Gapdh was used as a negative control. (C)Knockdown efficacy of Ezh2 siRNA in MIN6 cell lines was analyzed by qRT-PCR. si-Ezh2 group was a mixture of two interfering sequences. MIN6 cells (D) or primary islets (E) were transfected with mock (transfection agent alone), si-NC (a scrambled sequence), or si-Ezh2. qRT-PCR was used to detect the expressions of Ezh2, MafA and Ins2. (F) Western blot was used to analyze the expressions of EZH2 and MafA in MIN6 cells and pancreatic islets treated with mock, si-NC, or si-Ezh2 transfection; a representative of three experiments is shown. Beta actin protein was used as an internal control. ${ }^{* *}, \mathrm{P}<0.01{ }^{*}, \mathrm{P}<0.05$; two-tailed unpaired Student $\mathrm{t}$ test. Error bars, mean \pm SEM of three independent experiments.

the si-Ezh2 group was highest, this interference was adopted in the following experiments. The results were consistent with the inhibition of $M e g 3$ in previous studies. The expression levels of MafA and Ins2 (Meg3 primarily affects the expression of Ins2 [13])were significantly reduced by Ezh2 inhibition in both MIN6 cells and pancreatic islets (Fig. 1D, E). The same result for MafA was observed at the protein level (Fig. 1F). These findings indicate that lncRNA Meg3 regulates MafA expression through binding EZH2 in pancreatic beta cells.

\section{IncRNA Meg3 and EZH2 negatively regulate Rad21, Smc3, and Sin $3 \alpha$ expression}

It is clear that MafA is essential for the maintenance of islet structure and the function of beta cells $[2,12,39,40]$. The expression of Ins2 was significantly downregulated in MIN6 cells by MafA inhibition (Fig. 2A). The knockdown efficiency of MafA is shown in Fig. 2B; the si-MafA group was a mixture of two interfering sequences. The Caltech transcription factor binding site (TFBS), PSU TFBS, and Stan/Yale TFBS ChIP-sequences (http://genome.ucsc. edu/) showed binding of transcription factors to the MafA promoter region in various cells (Table 2). Based on the mechanism of EZH2, we needed to find the transcription factors that negatively regulate MafA. Thus, we inhibited Meg3 and Ezh2 in MIN6 cells and detected the expression of these transcription factors (Fig. 2C). The knockdown efficiency of Meg3 is shown in Fig. 2D; the si-Meg3 group was a mixture of two interfering sequences. The results show that the expression levels of Rad21, Smc3, and Sin $3 \alpha$ consistently increased in the two conditions. In addition, the same results were obtained after repeated experiments in pancreatic islet cells (Fig. 2E, F). Western blotting assays also confirmed that the expression levels of Rad21, Smc3, and Sin3 $\alpha$ were significantly higher compared to the control groups in MIN6 cells and pancreatic islets after knockdown of Meg3 or Ezh2 (Fig. 2G). These results suggest that Meg3 downregulated the expression of Rad21, Smc3, and Sin3 $\alpha$ in pancreatic beta cells through EZH2.

IncRNA Meg3 epigenetically regulates the expression of Rad21, Smc3, or Sin $3 \alpha$ by binding EZH2

To clarify the mechanism of Meg3 regulation of the three transcription factors, we examined whether EZH2 was bound to the promoter region of Rad21, Smc3 or $\operatorname{Sin} 3 \alpha$, 
Fig. 2. IncRNA Meg3 negative regulates $\operatorname{Rad} 21 / \mathrm{Smc} 3 / \mathrm{Sin} 3 \alpha$ expression. (A) qRT-PCR was used to detect the expressions of MafA and Ins2 in MIN6 cells treated with mock, si-NC, or siMafA, transfection. (B) Knockdown efficacy of MafA siRNA in MIN6 cell lines was analyzed by qRT-PCR. siMafA group was a mixture of two interfering sequences. (C) qRT-PCR was used to detect the expressions of transcription factors in MIN6 cells treated with mock, si-NC, si-Meg3, or si-Ezh2 transfection. (D) Knockdown efficacy of Meg3 siRNA in MIN6 cell lines was analyzed by qRT-PCR. si-Meg3 group was a mixture of two interfering se-

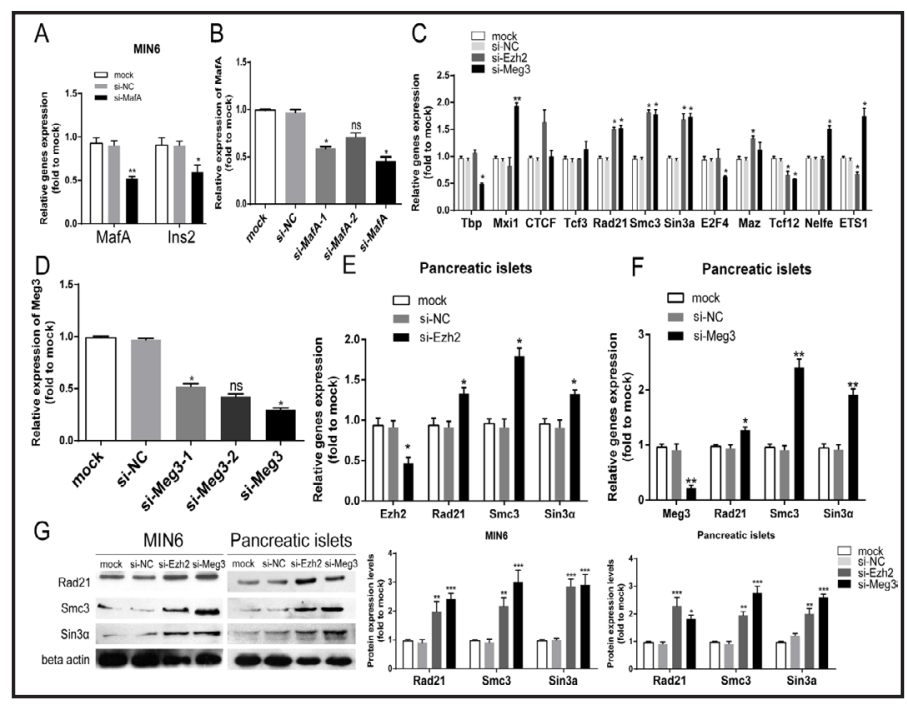
quences. (E)qRT-PCR analysis of the expressions of Ezh2, Rad21, Smc3, and Sin3 $\alpha$ in primary islets treated with mock, si-NC, or si-Ezh2 transfection was performed. (F) qRT-PCR analysis of the expressions of Meg3, Rad21, Smc3, and Sin3 $\alpha$ in primary islets treated with mock, si-NC, or si-Meg3 transfection was performed. (G) Protein levels of Rad21, Smc3, and Sin $3 \alpha$ in MIN6 cells and pancreatic islets treated with mock, si-NC, si-Ezh2, or si-Meg3 transfection were measured; a representative of three experiments is shown. Beta actin protein was used as an internal control. ${ }^{* *}, \mathrm{P}<0.001$; **, $\mathrm{P}<0.01{ }^{*}, \mathrm{P}<0.05$; two-tailed unpaired Student $\mathrm{t}$ test. Error bars, mean $\pm \mathrm{SEM}$ of three independent experiments.

whether IncRNA Meg3 was required for targeting EZH2, and the activity of the promoters of these transcription factors. As shown in Fig. 3, after transfection with siRNA of Meg3 for $48 \mathrm{~h}$ in MIN6 cells, EZH2 was bound to the promoters

Table 2. Transcription factors that bind to the MafA promoter region

\begin{tabular}{lc}
\hline Cell line & Transcription factor \\
\hline C2C12 & CTCF、E2F4、TCF12、TCF3 \\
CH12 & CTCF、ETS1、MAZ、 mxi1、NELFe、RAD21、SIN3A、SMC3、TBP \\
MEL & CTCF、ETS1、RAD21、SIN3A、SMC3、TBP
\end{tabular}
of Rad21, Smc3, and $\operatorname{Sin} 3 \alpha$, and we detected H3K27 trimethylation using ChIP-qPCR. Knockdown of Meg3 resulted in the loss of EZH2 binding (Fig. 3A-C) and H3K27 trimethylation occupancy of the Rad21, Smc3, and Sin $3 \alpha$ promoters (Fig. 3E-G), and no difference was detected at the promoter of Hoxa9, a known EZH2-targeted gene [41] (Fig. 3D, H). Our results suggest that IncRNA Meg3 binds to EZH2 via trimethylation of H3K27 in the Rad21, Smc3, and $\operatorname{Sin} 3 \alpha$ promoter, and in turn suppresses the expression of these transcription factors.

Rad21, Smc3 or Sin3 $\alpha$ can bind to the MafA promoter and negatively regulates MafA expression in pancreatic islets

Rad21 and Smc3, two core subunits of cohesin [42], are crucial for chromosome segregation and DNA repair [43, 44]. Sin $3 \alpha$ can recruit histone deacetylases (HDACs) to the proximal promoter and repress target gene expression $[45,46]$. However, there have been no reports regarding their regulation of MafA. It is known that Rad21, Smc3, and $\operatorname{Sin} 3 \alpha$ can bind to the promoter region of MafA in a variety of cells, but whether these transcription factors can bind to the MafA promoter in the islet cells is not clear. To determine whether they can bind to the MafA promoter region, we used ChIP-qPCR to analyze the process in MIN6 cells. The results show that Rad21, Smc3, and Sin $3 \alpha$ have a higher degree of promoter binding compared with the level of recruitment of IgG on the MafA promoter (Fig. 4A-C). This suggests that these three transcription factors can directly bind to the promoter region of MafA in pancreatic islet cells, and Meg3 silencing correlates with enhanced recruitment 


\section{Cellular Physiology and Biochemistry

Fig. 3. IncRNA Meg3 epigenetically regulates the expression of $\operatorname{Rad} 21 / \mathrm{Smc} 3 / \operatorname{Sin} 3 \alpha$ by binding EZH2. (A-D) ChIP-qPCR of EZH2 occupancy in the Rad21/ Smc3/Sin $3 \alpha /$ Hoxa9 promoters in MIN6 cells treated with si-NC or si-Meg3 was performed; IgG served as a negative control. (E-H) ChIP-qPCR of H3K27me3 binding in the Rad21/Smc3/Sin $3 \alpha /$ Hoxa9 promoters in MIN6 cells treated with si-NC or si-Meg3 was measured; IgG served as a negative control. *, P<0.05; twotailed unpaired Student $t$ test. Error bars, mean \pm SEM of three independent experiments.

of these transcription factors to the MafA promoter. To investigate whether $\operatorname{Rad} 21$, Smc3, and $\operatorname{Sin} 3 \alpha$ are transcriptional inhibitors of $M a f A$, we measured the expression of MafA after the inhibition of these genes in cells. After being transfected with specific siRNA of Rad21, Smc3, or Sin $3 \alpha$ in cells for 48 h, MafA expression was detected by qRT-PCR. We found that MafA expression was significantly increased in both MIN6 cells and pancreatic islets (Fig. 4D, E). Using western blotting assays, we also found that MafA protein level was upregulated by Rad21, Smc3, or Sin3 $\alpha$ inhibition (Fig. 4F). The knockdown efficiency of Rad21, Smc3, and $\operatorname{Sin} 3 \alpha$ is shown in Fig. 4G, J; the si-Rad21, si-
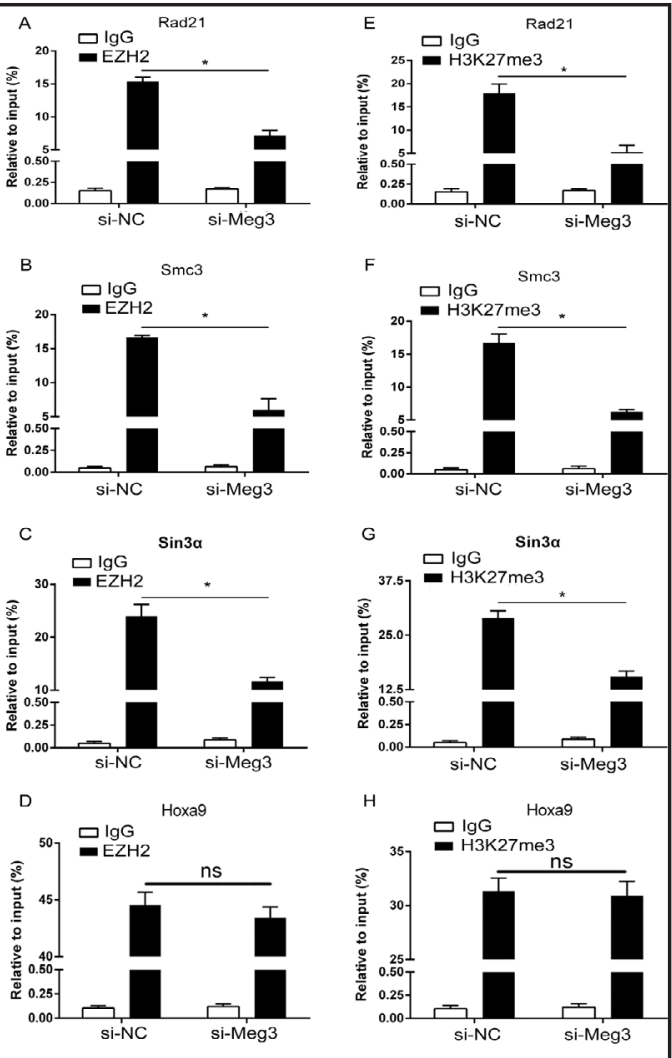

Smc3 and si-Sin $3 \alpha$ groups were a mixture of two interfering sequences. From these findings, we determined that Rad21, Smc3, or Sin3 $\alpha$ can inhibit the expression of MafA.

Suppression of Rad21, Smc3, or Sin3 $\alpha$ expression in MIN6 cells increases insulin synthesis and secretion through MafA

To determine whether Rad21, Smc3 or Sin $3 \alpha$ regulates the synthesis and secretion of insulin through MafA, we used siRNA targeting knockdown of Rad21, Smc3, or Sin3 $\alpha$ in MIN6 cells. After the transfection of siRNA into MIN6 cells for $48 \mathrm{~h}$, qRT-PCR analysis revealed that Ins2 expression was significantly increased in the si-Rad21, si-Smc3 and si-Sin $3 \alpha$ groups (Fig. 5A). Taking into account that the synthesis of insulin can affect insulin secretion [47], we detected GSIS of MIN6 cells after Rad21, Smc3, or Sin $3 \alpha$ inhibition. We found no significant changes in insulin release at a glucose level of $2 \mathrm{mM}$ but, compared with the control group, the secretion of insulin was markedly increased at a glucose level of $20 \mathrm{mM}$ after knockdown of Rad21, Smc3, or $\operatorname{Sin} 3 \alpha$ (Fig. 5B). The results show that the loss of Rad21, Smc3, or $\operatorname{Sin} 3 \alpha$ can promote the synthesis and secretion of insulin in MIN6 cells. Further, we analyzed whether Rad21, Smc3, and Sin3 $\alpha$ were abnormally expressed in diabetic mice. Primary islets were isolated from two types of diabetic mice, NOD and db/db. qRT-PCR analysis showed that the expression levels of Rad21, Smc3, and Sin $3 \alpha$ were increased in the two mouse models. By contrast, the expressions of Meg3, Ezh2, MafA, and Ins2 were reduced in diabetic pancreatic islets (Fig. 5C). Furthermore, after treating MIN6 cells with tumor necrosis factor alpha ( TNF $\alpha$ ) for $24 \mathrm{~h}$ and $48 \mathrm{~h}$, we obtained similar results to those observed in the two types of diabetic mice (Fig. 5D). These findings also suggest that Meg3 can influence MafA expression and insulin production by modulating $\operatorname{Rad} 21, \operatorname{Smc} 3$, or $\operatorname{Sin} 3 \alpha$ in mouse pancreatic islets.

Downregulation of Meg3 increases expression of Rad21, Smc3, and Sin3 $\alpha$ in vivo

We used the intravenous hydrodynamic method for administering siRNA in normal $\mathrm{Balb} / \mathrm{c}$ male mice. After isolation and purification of mouse pancreatic islets, we examined 
Fig. 4. $\operatorname{Rad} 21 / \operatorname{Smc} 3 / \operatorname{Sin} 3 \alpha$ can bind to the MafA promoter region and negatively regulate MafA expression in pancreatic islets. (A-C) ChIP-qPCR analysis of the capacity of $\operatorname{Rad} 21 / \mathrm{Smc} 3 / \mathrm{Sin} 3 \alpha$ binding to the MafA promoters in MIN6 cells was performed. qRT-PCR was used to detect the expressions of MafA in MIN6 cells (D) or primary islets (E) treated with mock, siNC, si-Rad21, si-Smc3 or si-Sin $3 \alpha$ transfection. (F) Protein levels of MafA in MIN6 cells and pancreatic islets treated with mock, siNC, si-Rad21, si-Smc3 or si-Sin $3 \alpha$ transfection were analyzed; a representative of three experiments is shown. Knockdown efficacy of Rad21, Smc3 and Sin3 $\alpha$ siRNA in MIN6 cell lines was analyzed by qRT-PCR (G-I) and western blot (J) ; a representative of three experi-
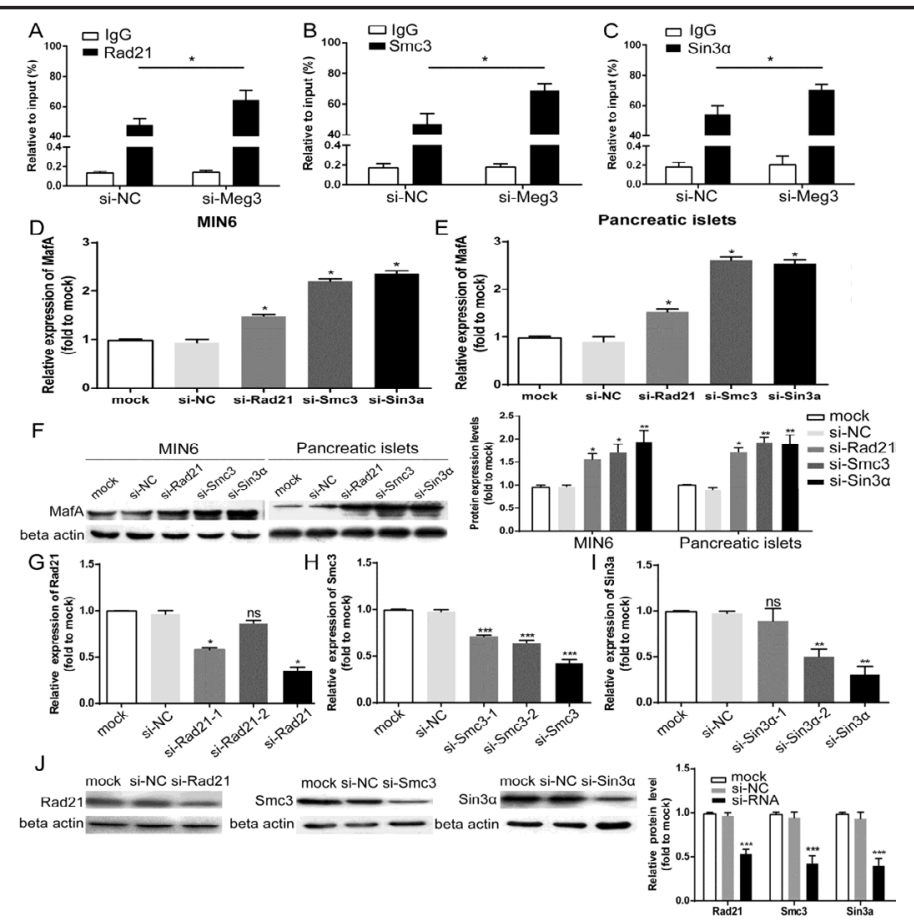
ments is shown. si-Rad21, si-Smc3

or si-Sin $3 \alpha$ group was a mixture of two interfering sequences. Beta actin protein was used as an internal control. ***, $\mathrm{P}<0.001$; ${ }^{* *}, \mathrm{P}<0.01 ;{ }^{*}, \mathrm{P}<0.05$; two-tailed unpaired Student $\mathrm{t}$ test. Error bars, mean $\pm \mathrm{SEM}$ of three independent experiments.

Fig. 5. Suppression of $\operatorname{Rad} 21 /$ Smc3/Sin $3 \alpha$ expression in MIN6 cells increases insulin synthesis and secretion. (A) qRT-PCR was used to detect the expressions of Ins2 in MIN6 cells treated with mock, si-NC, si-Rad21, si-Smc3 or si-Sin $3 \alpha$ transfection. (B) GSIS analysis of insulin secretion in response to 2 or $20 \mathrm{mM}$ glucose in MIN6 cells treated with mock, siNC, si-Rad21, si-Smc3, or si-Sin $3 \alpha$ transfection were performed. The

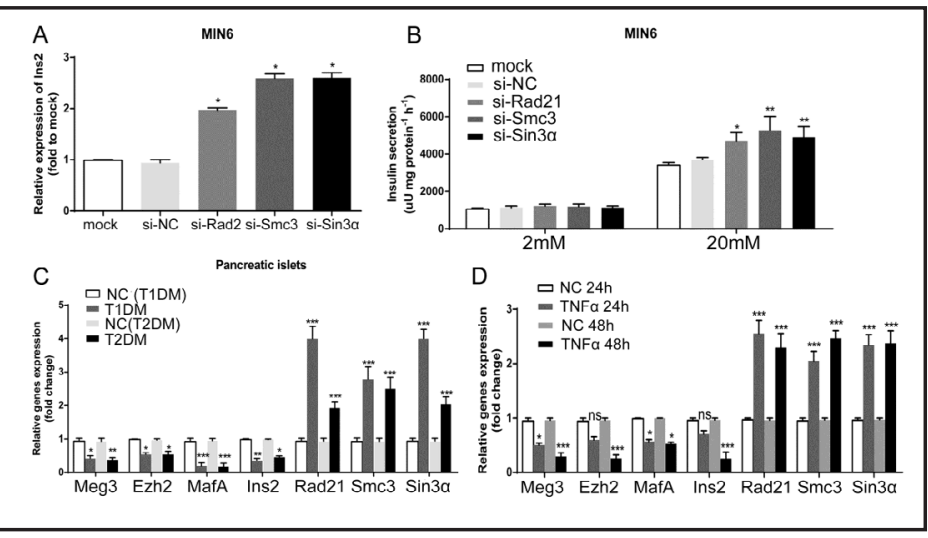
level of insulin was detected by RIA. (C) Meg3, Ezh2, MafA, Ins2, Rad21, Smc3, and Sin3 $\alpha$ were detected by qRT-PCR in islets isolated from T1DM or T2DM mice and compared with those from control mice. (D) Detection of genes expressions in MIN6 cells after being treated with TNF $\alpha$ for $24 \mathrm{~h}$ and $48 \mathrm{~h} .{ }^{* * *}, \mathrm{P}<0.001$; ${ }^{* *}, \mathrm{P}<0.01 ;{ }^{*}, \mathrm{P}<0.05$; two-tailed unpaired Student $t$ test. Error bars, mean \pm SEM of three independent experiments.

the expression of MafA, Rad21, Smc3, and $\operatorname{Sin} 3 \alpha$ by qRT-PCR and western blotting (Fig. 6A, B). The results showed that expression levels of Rad21, Smc3 and $\operatorname{Sin} 3 \alpha$ were increased by the inhibition of Meg3 in mouse pancreatic islets, and MafA expression was reduced at both the nucleic acid and protein levels. Similarly, the expression of Ins2 was significantly decreased at the nucleic acid level in vivo (Fig. 6A). These results further confirm our findings with the MIN6 cell line and mouse primary islet cells. 


\section{Cellular Physiology Cell Physiol Biochem 2018;45:2031-2043 \\ \begin{tabular}{l|l} 
and Biochemistry Published online: March 09, 2018 & $\begin{array}{l}\text { (c) } 2018 \text { The Author(s). Published by S. Karger AG, Basel } \\
\text { www.karger.com/cpb }\end{array}$ \\
\hline
\end{tabular} \\ Wang et al.: Pancreatic Meg3 Regulates MafA by Inactivating Rad21, Smc3 or $\operatorname{Sin} 3 \alpha$}

\section{Discussion}

Increasing evidence suggests that IncRNAs are associated with biological activities. lncRNAs have been shown to play a central role in physiology and human diseases [19], and numerous lncRNAs are expressed in a specific manner in different tissues and cells. With further research, lncRNAs have been shown to correlate with the function of pancreatic islets $[13,14]$ and the occurrence of diabetes $[15,16]$. However, there have been few studies on the molecular mechanism of IncRNAs in pancreatic islets.

In this study, we investigated the molecular mechanism of the lncRNA Meg3 and its influence on insulin synthesis by regulating the expression of the key transcription factor MafA in pancreatic beta cells. Pancreatic Meg3, encoded by the imprinted Dlk1-Meg3 gene region, was associated with the function of beta cells. According to the preliminary results, the expression of Meg3 was significantly downregulated in the islets of diabetic mice. Further, its deletion can result in the decrease of MafA and insulin in beta cells [13]. MafA, a key transcription factor, binds to the RIPE3b/C1 element of the insulin promoter [2] and principally regulates insulin biosynthesis and secretion [40,48]. Deficiency of MafA is closely related to the occurrence of diabetes $[49,50]$. Thus, we speculate that lncRNA Meg3 can influence the expression of insulin by regulating MafA. However, the molecular mechanism of Meg3 regulation of MafA in pancreatic islets is not clear.

An increasing number of studies have found that lncRNAs can regulate downstream target genes by binding to EZH2 in the nucleus. EZH2, a methyltransferase belonging to PRC2, can initiate trimethylation at lysine 27 in histone H3 [35]. Moreover, research has confirmed that MEG3 can regulate target gene expression by recruiting EZH2 in human breast cells [38]. Using PAR-CLIP, Kaneko et al. revealed that Meg3 can bind to EZH2 in mESCs. In addition, Meg3 RNA directs EZH2 to suppress transcription of Dlk1 in mESCs [51]. To clarify the mechanism of Meg3 regulation, we first determined the localization in pancreatic beta cells. By observing the subcellular localization, we found that Meg3 was primarily expressed in the nucleus and, as previously reported [37], can bind to EZH2 in MIN6 cells. Thus, pancreatic Meg3 can epigenetically inhibit the expression of target genes at the transcriptional level by binding EZH2 in the nucleus. Similar to the effects of Meg3 knockdown on MafA expression, the expression of MafA was reduced after inhibiting Ezh2 in MIN6 cells and pancreatic islets. According to the mechanism of Meg3 regulation of target genes through EZH2, we needed to find the negative transcription factors of MafA. Through the Caltech TFBS, PSU TFBS, and Stan/Yale TFBS ChIP-sequences, we found a number of transcription factors bound to the MafA promoter. After screening in MIN6 cells and pancreatic islets, we found that expression of Rad21, Smc3, and Sin $3 \alpha$ was negatively regulated by Meg3. Rad21 and Smc3 are two core subunits of cohesin, and are associated with chromosome segregation and DNA repair [43, 44]. Moreover, $\operatorname{Sin} 3 \alpha$ suppresses the expression of the target gene by recruiting HDACs to deacetylate histones $\mathrm{H} 3$ and $\mathrm{H} 4$ of the proximal promoter [45]. An intrauterine growth retardation state has been linked to the onset of type 2 diabetes in adulthood, along with the recruitment of $\operatorname{Sin} 3 \alpha$ and HDAC1 to the promoter of Pdx1 [46]. Through ChIP-qPCR analysis, we determined that Meg3 can act directly on the Rad21, Smc3, or Sin3 $\alpha$ promoter by binding EZH2, and inhibit the transcription of these genes by H3K27 trimethylation. We have demonstrated that IncRNA Meg3 can be directly responsible for the negative regulation of $\operatorname{Rad} 21, \operatorname{Smc} 3$, and $\operatorname{Sin} 3 \alpha$. However, whether they can negatively regulate MafA remains to be studied.

To confirm that Rad21, Smc3 and $\operatorname{Sin} 3 \alpha$ are negative regulators of MafA, we first investigated whether they were bound to the MafA promoter in pancreatic beta cells. ChIPqPCR analysis was used to detect the recruitment of Rad21, Smc3, or Sin3 $\alpha$ in the MafA promoter region, and we found that the recruitment of the three transcription factors in the MafA promoter was significantly higher than in the IgG group in MIN6 cells. In addition, Meg3 silencing correlated with enhanced recruitment of Rad21, Smc3 or Sin3 $\alpha$ to the MafA promoter. These results indicate that Rad21, Smc3 or $\operatorname{Sin} 3 \alpha$ can also be directly bound to the promoter of MafA in mouse beta cells. Additionally, as the expression of Meg3 is 


\section{Cellular Physiology Cell Physiol Biochem 2018;45:2031-2043 and Biochemistry \begin{tabular}{c|c} 
DOI: 10.1159/000487983 \\
Published ontrne:Tarch 09, 2018 & $\begin{array}{l}\text { O 2018 The Author(s). Published by S. Karger AG, Basel } \\
\text { www.karger.com/cpb }\end{array}$
\end{tabular}

Fig. 6. Downregulation of IncRNA Meg3 increases expression of Rad21/ Smc3/Sin $3 \alpha$ in vivo. Normal Balb/c male mice were injected with PBS (mock), scramble siRNA (si-NC) or Meg3 siRNA (si-Meg3) by intravenous hydrodynamic method, and mouse pancreatic islets were collected after injection 48 h. Expression of MafA, Rad21, Smc3, and Sin3 $\alpha$ in pancreatic islets were detected by qRT-PCR (A) and western blot (B); a representative of three experiments is shown. Ins2 expression was detected by qRT-PCR (A). Beta actin protein was used as an internal control. ***, $\mathrm{P}<0.001$; **, $\mathrm{P}<0.01$; *, $\mathrm{P}<0.05$; two-tailed unpaired Student $t$ test. Error bars, mean $\pm \mathrm{SEM}$ of three independent experiments.

downregulated, the recruitment of these transcription factors on the MafA promoter is enhanced. To prove that $\operatorname{Rad} 21, \operatorname{Smc} 3$, or $\operatorname{Sin} 3 \alpha$ can negatively regulate the expression of MafA, we interfered with the expression of these transcription factors in MIN6 cells and pancreatic islets by siRNA, and then determined the expression of MafA at both the nucleic acid and protein levels. If Rad21, Smc 3 and $\operatorname{Sin} 3 \alpha$ negatively regulate the expression of MafA, their inhibition will promote the expression of MafA. The subsequent results confirmed our hypothesis. By specific interference of these transcription factors, the expression of MafA was upregulated in MIN6 cells and pancreatic islet cells. Moreover, in the rescue experiment, the expression of MafA was downregulated by inhibition of $M e g 3$, and subsequent to the inhibition of Ras21, Smc3, or Sin3 $\alpha$ in MIN6 cells, the expression of MafA was restored at the nucleic acid and protein levels (data not shown). MafA is an important transcription factor regulating the expression of insulin. Upregulated expression of MafA can promote the synthesis and secretion of insulin, and play a pivotal role in the function of islet cells. We believe that if $\operatorname{Rad} 21, \operatorname{Smc} 3$ or $\operatorname{Sin} 3 \alpha$ negatively regulates the expression of MafA, then inhibiting their expression, which promotes the upregulated expression of MafA, should also promote the synthesis and secretion of insulin. Therefore, we examined the synthesis and secretion of insulin by inhibiting Rad21, Smc3, or Sin $3 \alpha$ in MIN6 cells. We found that the expression of insulin was increased after interfering with these transcription factors, which was consistent with the expression of MafA. In addition, insulin secretion was higher in the presence of $20 \mathrm{mM}$ glucose after Rad21, Smc3, or Sin $3 \alpha$ knockdown compared with the control in MIN6 cells. Therefore, the experimental results are consistent with our proposal; after the suppression of these transcription factors, the synthesis and secretion of insulin was significantly increased. Thus, we determined the negative regulatory effect of Rad21, Smc3, and $\operatorname{Sin} 3 \alpha$ on MafA in mouse pancreatic islets, and their effect on the synthesis and secretion of insulin by inhibiting MafA.

Overall, our findings show the molecular mechanism of lncRNA Meg3 in regulating MafA in pancreatic beta cells. Pancreatic Meg3 suppresses the MafA negative regulating transcription factors Rad21, Smc3 or Sin $3 \alpha$ by methylation of EZH2, which upregulates the expression of MafA, and promotes the synthesis and secretion of insulin. Interestingly, in two types of diabetic mice (NOD and db/db), the expression of these transcription factors was significantly increased, whereas the expressions of MafA, Ins2, Meg3, and Ezh2 were markedly decreased. After treating MIN6 cells with TNF $\alpha$, a major pro-inflammatory cytokine associated with the occurrence of diabetes, the expression of these genes was measured and the results were consistent with the two types of diabetic mice. In addition, we validated the results observed in cells in vivo and obtained consistent results. These results confirm that 


\section{Cellular Physiology Cell Physiol Biochem 2018;45:2031-2043

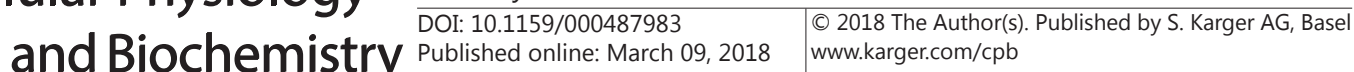

Meg3 plays an important role in the function of mouse islets, by binding EZH2 to regulate downstream target genes, thus affecting the synthesis of insulin.

\section{Conclusion}

We have demonstrated the mechanism of Meg3 regulation of pancreatic beta cells function. Our findings add to the current understanding of the regulatory mechanism of islet cell function and the possible mechanism of the development of diabetes. We will assess whether the same mechanisms operate in diabetic mice and humans in future studies.

\section{Acknowledgements}

The authors thank Xuezhi He, Hongwei Ma, Lianghui You for skilled technical assistance. This study was supported by the National Natural Science Foundation of China (No. 81570697), Open Research Project of State Key Laboratory of Reproductive Medicine (SKLRM-KF-1201).

NW conceived the experiments, generated the research, analysed the data, wrote the manuscript and approved its final version. YZ contributed to the acquisition of data, reviewed and edited the manuscript. MX, LW, FJ collected and analysed the data. QY and WD conceived the experiments, analysed the research data, critical revision of the manuscript and approved its final version. All authors have approved the final version and NW is responsible for the integrity of the work as a whole.

\section{Disclosure Statement}

The authors declare that there is not Disclosure Statement that would prejudice the impartiality of the reported research.

\section{References}

1 Gu G, Wells JM, Dombkowski D, Preffer F, Aronow B, Melton DA: Global expression analysis of gene regulatory pathways during endocrine pancreatic development. Development 2004;131:165-179.

2 Olbrot M, Rud J, Moss LG, Sharma A: Identification of beta-cell-specific insulin gene transcription factor RIPE3b1 as mammalian MafA. Proc Natl Acad Sci U S A 2002;99:6737-6742.

- Kataoka K, Han SI, Shioda S, Hirai M, Nishizawa M, Handa H: MafA is a glucose-regulated and pancreatic beta-cell-specific transcriptional activator for the insulin gene. J Biol Chem 2002;277:49903-49910.

4 Matsuoka TA, Zhao L, Artner I, Jarrett HW, Friedman D, Means A, Stein R: Members of the large Maf transcription family regulate insulin gene transcription in islet beta cells. Mol Cell Biol 2003;23:6049-6062.

5 Matsuoka TA, Kaneto H, Miyatsuka T, Yamamoto T, Yamamoto K, Kato K, Shimomura I, Stein R, Matsuhisa M: Regulation of MafA expression in pancreatic beta-cells in $\mathrm{db} / \mathrm{db}$ mice with diabetes. Diabetes 2010;59:1709-1720.

6 Naya FJ, Huang HP, Qiu Y, Mutoh H, DeMayo FJ, Leiter AB, Tsai MJ: Diabetes, defective pancreatic morphogenesis, and abnormal enteroendocrine differentiation in BETA2/neuroD-deficient mice. Genes Dev 1997;11:2323-2334.

7 Glick E, Leshkowitz D, Walker MD: Transcription factor BETA2 acts cooperatively with E2A and PDX1 to activate the insulin gene promoter. J Biol Chem 2000;275:2199-2204.

8 Ohlsson H, Karlsson K, Edlund T: IPF1, a homeodomain-containing transactivator of the insulin gene. EMBO J 1993;12:4251-4259. 


\section{Cellular Physiology Cell Physiol Biochem 2018;45:2031-2043 \begin{tabular}{l|l|l} 
DOI: 10.1159/000487983 & (c) 2018 The Author(s). Published by S. Karger AG, Basel
\end{tabular} and Biochemistry

- Leonard J, Peers B, Johnson T, Ferreri K, Lee S, Montminy MR: Characterization of somatostatin transactivating factor-1, a novel homeobox factor that stimulates somatostatin expression in pancreatic islet cells. Mol Endocrinol 1993;7:1275-1283.

10 Artner I, Hang Y, Mazur M, Yamamoto T, Guo M, Lindner J, Magnuson MA, Stein R: MafA and MafB regulate genes critical to beta-cells in a unique temporal manner. Diabetes 2010;59:2530-2539.

-11 Nishimura W, Kondo T, Salameh T, El Khattabi I, Dodge R, Bonner-Weir S, Sharma A: A switch from MafB to MafA expression accompanies differentiation to pancreatic beta-cells. Dev Biol 2006;293:526-539.

$\$ 12$ Nishimura W, Takahashi S, Yasuda K: MafA is critical for maintenance of the mature beta cell phenotype in mice. Diabetologia 2015;58:566-574.

13 You L, Wang N, Yin D, Wang L, Jin F, Zhu Y, Yuan Q, De W: Downregulation of Long Noncoding RNA Meg3 Affects Insulin Synthesis and Secretion in Mouse Pancreatic Beta Cells. J Cell Physiol 2015

14 Yin DD, Zhang EB, You LH, Wang N, Wang LT, Jin FY, Zhu YN, Cao LH, Yuan QX, De W, Tang W: Downregulation of lncRNA TUG1 affects apoptosis and insulin secretion in mouse pancreatic beta cells. Cell Physiol Biochem 2015;35:1892-1904.

-15 Kameswaran V, Bramswig NC, McKenna LB, Penn M, Schug J, Hand NJ, Chen Y, Choi I, Vourekas A, Won KJ, Liu C, Vivek K, Naji A, Friedman JR, Kaestner KH: Epigenetic regulation of the DLK1-MEG3 microRNA cluster in human type 2 diabetic islets. Cell Metab 2014;19:135-145.

-16 Moran I, Akerman I, van de Bunt M, Xie R, Benazra M, Nammo T, Arnes L, Nakic N, Garcia-Hurtado J, Rodriguez-Segui S, Pasquali L, Sauty-Colace C, Beucher A, Scharfmann R, van Arensbergen J, Johnson PR, Berry A, Lee C, Harkins T, Gmyr V, Pattou F, Kerr-Conte J, Piemonti L, Berney T, Hanley N, Gloyn AL, Sussel L, Langman L, Brayman KL, Sander M, McCarthy MI, Ravassard P, Ferrer J: Human beta cell transcriptome analysis uncovers IncRNAs that are tissue-specific, dynamically regulated, and abnormally expressed in type 2 diabetes. Cell Metab 2012;16:435-448.

-17 Spizzo R, Almeida MI, Colombatti A, Calin GA: Long non-coding RNAs and cancer: a new frontier of translational research? Oncogene 2012;31:4577-4587.

-18 Tsai MC, Spitale RC, Chang HY: Long intergenic noncoding RNAs: new links in cancer progression. Cancer Res 2011;71:3-7.

19 Guttman M, Rinn JL: Modular regulatory principles of large non-coding RNAs. Nature 2012;482:339-346.

20 Nogueira TC, Paula FM, Villate O, Colli ML, Moura RF, Cunha DA, Marselli L, Marchetti P, Cnop M, Julier C, Eizirik DL: GLIS3, a susceptibility gene for type 1 and type 2 diabetes, modulates pancreatic beta cell apoptosis via regulation of a splice variant of the BH3-only protein Bim. PLoS Genet 2013;9:e1003532.

-21 Miyoshi N, Wagatsuma H, Wakana S, Shiroishi T, Nomura M, Aisaka K, Kohda T, Surani MA, Kaneko-Ishino T, Ishino F: Identification of an imprinted gene, Meg3/Gtl2 and its human homologue MEG3, first mapped on mouse distal chromosome 12 and human chromosome 14q. Genes Cells 2000;5:211-220.

-22 Schuster-Gossler K, Simon-Chazottes D, Guenet JL, Zachgo J, Gossler A: Gtl2lacZ, an insertional mutation on mouse chromosome 12 with parental origin-dependent phenotype. Mamm Genome 1996;7:20-24.

-23 Dorrell C, Schug J, Lin CF, Canaday PS, Fox AJ, Smirnova O, Bonnah R, Streeter PR, Stoeckert CJ, Jr., Kaestner KH, Grompe M: Transcriptomes of the major human pancreatic cell types. Diabetologia 2011;54:28322844.

-24 Wallace C, Smyth DJ, Maisuria-Armer M, Walker NM, Todd JA, Clayton DG: The imprinted DLK1-MEG3 gene region on chromosome 14q32.2 alters susceptibility to type 1 diabetes. Nat Genet 2010;42:68-71.

-25 Xu G, Chen J, Jing G, Shalev A: Thioredoxin-interacting protein regulates insulin transcription through microRNA-204. Nat Med 2013;19:1141-1146.

26 You L, Wang N, Yin D, Wang L, Jin F, Zhu Y, Yuan Q De W: Downregulation of Long Noncoding RNA Meg3 Affects Insulin Synthesis and Secretion in Mouse Pancreatic Beta Cells. J Cell Physiol 2016;231:852-862.

27 Sun M, Xia R, Jin F, Xu T, Liu Z, De W, Liu X: Downregulated long noncoding RNA MEG3 is associated with poor prognosis and promotes cell proliferation in gastric cancer. Tumour Biol 2014;35:1065-1073.

28 Poy MN, Hausser J, Trajkovski M, Braun M, Collins S, Rorsman P, Zavolan M, Stoffel M: miR-375 maintains normal pancreatic alpha- and beta-cell mass. Proc Natl Acad Sci U S A 2009;106:5813-5818.

29 Liew CW, Bochenski J, Kawamori D, Hu J, Leech CA, Wanic K, Malecki M, Warram JH, Qi L, Krolewski AS, Kulkarni RN: The pseudokinase tribbles homolog 3 interacts with ATF4 to negatively regulate insulin exocytosis in human and mouse beta cells. J Clin Invest 2010;120:2876-2888.

-30 Lin Y, Sun Z: Antiaging gene Klotho enhances glucose-induced insulin secretion by up-regulating plasma membrane levels of TRPV2 in MIN6 beta-cells. Endocrinology 2012;153:3029-3039. 


\section{Cellular Physiology Cell Physiol Biochem 2018;45:2031-2043 \begin{tabular}{ll|l} 
DOI: 10.1159/000487983 & Ond Biochemistry 2018 The Author(s). Published by S. Karger AG, Basel \\
Published online: March 09, 2018 & \begin{tabular}{l} 
www.karger.com/cpb \\
\cline { 2 - 3 }
\end{tabular}
\end{tabular}}

-31 Benetatos L, Voulgaris E, Vartholomatos G, Hatzimichael E: Non-coding RNAs and EZH2 interactions in cancer: long and short tales from the transcriptome. Int J Cancer 2013;133:267-274.

-32 Luo M, Li Z, Wang W, Zeng Y, Liu Z, Qiu J: Long non-coding RNA H19 increases bladder cancer metastasis by associating with EZH2 and inhibiting E-cadherin expression. Cancer Lett 2013;333:213-221.

33 Hirata H, Hinoda Y, Shahryari V, Deng G, Nakajima K, Tabatabai ZL, Ishii N, Dahiya R: Long Noncoding RNA MALAT1 Promotes Aggressive Renal Cell Carcinoma through Ezh2 and Interacts with miR-205 Cancer Res 2015;75:1322-1331.

-34 Zhang EB, Kong R, Yin DD, You LH, Sun M, Han L, Xu TP, Xia R, Yang JS, De W, Chen J: Long noncoding RNA ANRIL indicates a poor prognosis of gastric cancer and promotes tumor growth by epigenetically silencing of miR-99a/miR-449a. Oncotarget 2014;5:2276-2292.

-35 Cao R, Wang L, Wang H, Xia L, Erdjument-Bromage H, Tempst P, Jones RS, Zhang Y: Role of histone H3 lysine 27 methylation in Polycomb-group silencing. Science 2002;298:1039-1043.

-36 Kaneko S, Son J, Shen SS, Reinberg D, Bonasio R: PRC2 binds active promoters and contacts nascent RNAs in embryonic stem cells. Nat Struct Mol Biol 2013;20:1258-1264.

-37 Kaneko S, Bonasio R, Saldana-Meyer R, Yoshida T, Son J, Nishino K, Umezawa A, Reinberg D: Interactions between JARID2 and noncoding RNAs regulate PRC2 recruitment to chromatin. Mol Cell 2014;53:290-300.

38 Mondal T, Subhash S, Vaid R, Enroth S, Uday S, Reinius B, Mitra S, Mohammed A, James AR, Hoberg E, Moustakas A, Gyllensten U, Jones SJ, Gustafsson CM, Sims AH, Westerlund F, Gorab E, Kanduri C: MEG3 long noncoding RNA regulates the TGF-beta pathway genes through formation of RNA-DNA triplex structures. Nat Commun 2015;6:7743.

39 Barrow J, Hay CW, Ferguson LA, Docherty HM, Docherty K: Transcription factor cycling on the insulin promoter. FEBS Lett 2006;580:711-715.

-40 Zhang C, Moriguchi T, Kajihara M, Esaki R, Harada A, Shimohata H, Oishi H, Hamada M, Morito N, Hasegawa K, Kudo T, Engel JD, Yamamoto M, Takahashi S: MafA is a key regulator of glucose-stimulated insulin secretion. Mol Cell Biol 2005;25:4969-4976.

41 Cao R, Zhang Y: SUZ12 is required for both the histone methyltransferase activity and the silencing function of the EED-EZH2 complex. Mol Cell 2004;15:57-67.

42 Hagstrom KA, Meyer BJ: Condensin and cohesin: more than chromosome compactor and glue. Nat Rev Genet 2003;4:520-534.

43 Supernat A, Lapinska-Szumczyk S, Sawicki S, Wydra D, Biernat W, Zaczek AJ: Deregulation of RAD21 and RUNX1 expression in endometrial cancer. Oncol Lett 2012;4:727-732.

44 Schmiesing JA, Ball AR, Jr., Gregson HC, Alderton JM, Zhou S, Yokomori K: Identification of two distinct human SMC protein complexes involved in mitotic chromosome dynamics. Proc Natl Acad Sci U S A 1998;95:12906-12911.

45 Hassig CA, Fleischer TC, Billin AN, Schreiber SL, Ayer DE: Histone deacetylase activity is required for full transcriptional repression by mSin3A. Cell 1997;89:341-347.

-46 Park JH, Stoffers DA, Nicholls RD, Simmons RA: Development of type 2 diabetes following intrauterine growth retardation in rats is associated with progressive epigenetic silencing of Pdx1 J Clin Invest 2008;118:2316-2324.

-47 Takamoto I, Kubota N, Nakaya K, Kumagai K, Hashimoto S, Kubota T, Inoue M, Kajiwara E, Katsuyama H, Obata A, Sakurai Y, Iwamoto M, Kitamura T, Ueki K, Kadowaki T: TCF7L2 in mouse pancreatic beta cells plays a crucial role in glucose homeostasis by regulating beta cell mass. Diabetologia 2014;57:542-553.

48 Wang H, Brun T, Kataoka K, Sharma AJ, Wollheim CB: MAFA controls genes implicated in insulin biosynthesis and secretion. Diabetologia 2007;50:348-358.

49 Noso S, Kataoka K, Kawabata Y, Babaya N, Hiromine Y, Yamaji K, Fujisawa T, Aramata S, Kudo T, Takahashi S, Ikegami H: Insulin transactivator MafA regulates intrathymic expression of insulin and affects susceptibility to type 1 diabetes. Diabetes 2010;59:2579-2587.

50 Butler AE, Robertson RP, Hernandez R, Matveyenko AV, Gurlo T, Butler PC: Beta cell nuclear musculoaponeurotic fibrosarcoma oncogene family A (MafA) is deficient in type 2 diabetes. Diabetologia 2012;55:2985-2988.

-51 Zhao J, Ohsumi TK, Kung JT, Ogawa Y, Grau DJ, Sarma K, Song JJ, Kingston RE, Borowsky M, Lee JT: Genomewide identification of polycomb-associated RNAs by RIP-seq. Mol Cell 2010;40:939-953. 